\title{
REPRESENTATIONS OF COMPACT GROUPS ON TOPOLOGICAL VECTOR SPACES: SOME REMARKS
}

\author{
RUSSELL A. JOHNSON
}

\begin{abstract}
A standard theorem concerning the decomposition of a representation of a compact group on a Hilbert space $E$ is generalized to the case when $E$ is locally convex and quasi-complete. As a corollary, it is shown that if $E$ is topologically irreducible, then it is finite dimensional.
\end{abstract}

1. Introduction. Let a compact group $G$ be represented unitarily on a Hilbert space $H$. Then there is a "canonical" decomposition $H=\bigoplus_{\sigma \in \hat{G}} H_{\sigma}$, where the invariant subspaces $H_{\sigma}$ are indexed by the elements $\sigma$ of $\hat{G}$, the set of equivalence classes of irreducible unitary representations of $G$. Each subspace $H_{\sigma}$ is characterized as the smallest subspace of $H$ containing all invariant subspaces $V$ of type $\sigma$ ( $V$ is of type $\sigma$ if the representation of $G$ on $V$ is in $\sigma$ ). Each $H_{\sigma}$ may be expressed (nonuniquely) as a direct sum of invariant subspaces $H_{\sigma, i}$ of type $\sigma$.

We here replace $H$ by a quasi-complete topological vector space $E$. Analogues of the above statements are proven. It is also shown that if $E$ is topologically irreducible, then $E$ has finite dimension.

\section{Preliminaries.}

2.1. Notation. We let $E$ denote a Hausdorff, locally convex, quasi-complete (closed bounded sets are complete) topological vector space, $G$ a compact topological group which is represented continuously on $E$. Thus there is a continuous map $(g, e) \rightarrow g \cdot e: G \times E \rightarrow E$ such that each map $g: e \rightarrow g \cdot e$ is linear. The notation $(G, E)$ indicates that $G$ is represented continuously on $E$. We use $d g, d g^{\prime}$, etc. to refer to normalized Haar measure on $G$.

2.2. Definitions. Let $\hat{G}$ be the set of equivalence classes of irreducible unitary representations of $G[2,27.3]$. If $\sigma \in \hat{G}$, let $d_{\sigma}$ be the dimension of $V$ for some (hence any) $(G, V) \in \sigma$.

2.3. Definition. A representation $(G, E)$ is topologically irreducible if $E$ contains no proper closed $G$-invariant subspaces except $\{0\}$.

We review the definition and certain basic properties of the weak integral; , for more details see [1, Chapter III, §§3-4].

2.4. Let $X$ be a locally compact topological space. Let $K(X)=\{f: X \rightarrow$ $C \mid f$ is continuous with compact support $\}, K(X, E)=\{f: X \rightarrow E \mid f$ is continuous with compact support $\}$. Let $E^{\prime}$ be the topological dual of $E$ (continu-

Received by the editors January 1, 1976.

AMS (MOS) subject classifications (1970). Primary 22C05, 28A45.

Copyright $\odot 1977$, American Mathematical Society 
ous linear functionals), $E^{\prime *}$ the algebraic dual of $E^{\prime}$ (all linear functionals). Let $\mu$ be a measure on $X$ (a continuous linear functional on $(K(X), \mathscr{T})$, $\mathcal{T}=$ topology of uniform convergence on compact sets). If $f \in K(X, E)$, define the weak integral of $f$ with respect to $\mu$ as follows:

$$
\left\langle e^{\prime}, \int_{X} f(x) d \mu(x)\right\rangle=\int_{X}\left\langle e^{\prime}, f(x)\right\rangle d \mu(x) \quad\left(e^{\prime} \in E^{\prime}\right) .
$$

2.5. TheOREM. (a) If $f \in K(X, E)$, then $\int_{X} f(x) d \mu(x) \in E$.

(b) If $q$ is a continuous seminorm on $E$, then $q\left(\int_{X} f(x) d \mu(x)\right) \leqslant$. $\int_{X} q(f(x)) d \mu(x)$.

(c) If $Y$ is another locally compact space, $\nu$ a measure on $Y$, and

$$
f \in K(X \times Y, E),
$$

then

$$
\begin{aligned}
\int_{X \times Y} f(x, y) d \mu(x) d \nu(y) & =\int_{X} d \mu(x)\left[\int_{Y} f(x, y) d \nu(y)\right] \\
& =\int_{Y} d \nu(y)\left[\int_{X} f(x, y) d \mu(x)\right] .
\end{aligned}
$$

For the proofs see [1, Chapter III: $\S 3$, No. 3, Corollary 2; $\$ 3$, No. 2, Proposition 6; $\S 3$, No. 2, Proposition 2; $\S 4$, No. 1, Remark following Theorem 2].

2.6. Let $M(G)$ be the convolution algebra $[2,19.12]$ of $G$; denote convolution by $*$. The action of $G$ on $E$ extends to an algebra homomorphism $U$ from $M(G)$ to $L(E)$, the continuous linear operators on $E$; the extension is given by $U(\nu) \cdot e=\int_{G}(g \cdot e) d \nu(g)$. For a proof of this, see the two paragraphs preceding 4.1.1.2 in [3]; note that (i) $G$ is compact, so all measures on $G$ have compact support; (ii) the proof uses only quasi-completeness, not completeness, of $E$.

2.7. Definitions. Let $\sigma \in \hat{G},(G, V) \in \sigma$. Let $v_{1}, \ldots, v_{n}\left(n=d_{\sigma}\right)$ be an orthonormal basis in $V$, and let $u_{i j}^{o}(g)=\left\langle g v_{j}, v_{i}\right\rangle(g \in G, 1 \leqslant i, j \leqslant n)$, where $\langle$,$\rangle is the inner product in V$. Let $\mathcal{T}_{\sigma}(G)$ be the linear span in $C(G)$ of the functions $u_{i j}^{\sigma}$, and let $\mathcal{T}(G)$ be the span in $C(G)$ of $\cup_{\sigma \in \hat{G}} \mathcal{T}_{\sigma}(G)$. Let $\lambda_{\sigma}(g)=\sum_{i=1}^{n} u_{i i}^{\sigma}(g)(g \in G) ; \lambda_{\sigma}$ is the character of $\sigma$. See [2, 27.5-27.8].

2.8. THEOREM. (a) (Orthogonality) $\int_{G} u_{i j}^{\sigma}(g) u_{k l}^{\tau}(g) d g=\delta_{i k} \delta_{j l} \delta_{\sigma \tau}(\sigma, \tau \in \hat{G})$.

(b) (Peter-Weyl theorem) $\mathcal{T}(G)$ is uniformly dense in $C(G)$.

(c) If $\sigma, \tau \in \hat{G}$ and $\phi \in \mathcal{T}_{\sigma}(G)$, then $\phi * d_{\sigma} \lambda_{\sigma}=d_{\sigma} \lambda_{\sigma} * \phi=\phi$.

(d) If $\sigma, \tau \in \hat{G}$, then $\lambda_{\sigma} * \lambda_{\tau}=\delta_{\sigma \tau} \lambda_{\sigma} / d_{\sigma}$.

For the proofs, see $[2,27.19 ; 27.40 ; 27.24($ ii); $27.24($ iii)]

2.9. Definition. Let $(G, E)$ be a representation, $E$ quasi-complete. Say that $(G, E)$ is of type $\sigma$ if for some (hence any) $(G, V) \in \sigma$, there is a linear isomorphism $W: V \rightarrow E$ such that $W(g \cdot v)=g \cdot W(v)$. (Thus $W$ intertwines $(G, E)$ and $(G, V)$.) Abusing notation, we write $(G, E) \in \sigma$. 
3. Results. Recall that a locally convex topology is determined by its set of continuous seminorms.

3.1. Definition. A seminorm $p$ on $E$ is $G$-invariant if $p(g \cdot e)=p(e)$ $(g \in G, e \in E)$.

3.2. Lemma. The topology on $E$ is determined by the set of continuous $G$-invariant seminorms.

Proof. Let $q$ be a continuous seminorm on $E$. It suffices to show that there is a continuous $G$-invariant seminorm $p$ such that $q(e) \leqslant p(e)(e \in E)$. To prove this, let $p(e)=\sup _{g \in G} q(g e) ; p$ is an invariant seminorm. To demonstrate continuity of $p$, let $e_{n} \rightarrow 0$ (a seminorm on $E$ is continuous iff it is continuous at 0 ). Let $\varepsilon>0$ be given, and let $U_{\varepsilon}=\{e \in E \mid q(e)<\varepsilon\}$. Since $G$ is compact, it acts equicontinuously on $E$; let $V$ be a neighborhood of 0 such that $G \cdot V \subset U_{\varepsilon}$. If $n>n_{0} \Rightarrow e_{n} \in V$, then $n \geqslant n_{0} \Rightarrow p\left(e_{n}\right)=\sup _{g \in G} q\left(g e_{n}\right)$ $<\varepsilon$. So $p$ is continuous.

Let $\sigma \in \hat{G}$; we define an operator $P_{\sigma}: E \rightarrow E$ as follows:

$$
P_{\sigma}(e)=d_{\sigma} \int_{G} \overline{\lambda_{\sigma}(g)}(g e) d g,
$$

where $\lambda_{\sigma}$ is the appropriate character (2.7). By 2.5(a), $P_{\sigma}$ is well defined; it is easily seen to be linear.

3.3. Proposition. (a) $P_{\sigma}$ is continuous $(\sigma \in \hat{G})$.

(b) $P_{\sigma} P_{\tau}=P_{\tau} P_{\sigma}=0(\sigma \neq \tau) ; P_{\sigma}^{2}=P_{\sigma}$.

(c) $g P_{\sigma}=P_{\sigma} g(g \in G)$.

Proof. In the proof, we make free use of 2.5(a)-(d).

(a) Let $e_{n} \rightarrow 0$ and suppose $p$ is a continuous $G$-invariant seminorm. Then $p\left(P_{\sigma}\left(e_{n}\right)\right)=d_{\sigma} p\left(\int_{G} \overline{\lambda_{\sigma}(g)}\left(g e_{n}\right) d g\right) \leqslant d_{\sigma} \int_{G}\left|\overline{\lambda_{\sigma}(g)}\right| p\left(g e_{n}\right) d g \leqslant d_{\sigma}^{2} p\left(e_{n}\right) \rightarrow 0$.

By $3.1, P_{\sigma}$ is continuous.

(b) Note that

$$
\begin{aligned}
P_{\sigma}\left(P_{\tau}(e)\right) & =d_{\sigma}^{2} \int_{G} \overline{\lambda_{\sigma}(g)} g \cdot\left[\int_{G} \overline{\lambda_{\tau}\left(g^{\prime}\right)}\left(g^{\prime} e\right) d g^{\prime}\right] d g \\
& =d_{\sigma}^{2} \int_{G} \int_{G} \overline{\lambda_{\tau}(g)} \overline{\lambda_{\sigma}\left(g^{\prime}\right)}\left(g g^{\prime} e\right) d g^{\prime} d g \\
& =d_{\sigma}^{2} \int_{G} \tilde{g} e\left[\int_{G} \overline{\lambda_{\sigma}(g)} \overline{\lambda_{\tau}\left(g^{-1} \tilde{g}\right)} d g\right] \\
& =d_{\sigma}^{2} \int_{G}(\tilde{g} e) \overline{\lambda_{\sigma} * \lambda_{\tau}(\tilde{g})} d \tilde{g} .
\end{aligned}
$$

Since $\lambda_{\sigma} * \lambda_{\tau}=\delta_{\sigma \tau} \lambda_{\sigma} / d_{\sigma}$, the last integral is zero if $\sigma \neq \tau$, and is $d_{\sigma} \int_{G}(\tilde{g} e) \overline{\lambda_{\sigma}(\tilde{g})} d \tilde{g}=P_{\sigma}(e)$ if $\sigma=\tau$.

(c) We compute: 


$$
\begin{aligned}
g \cdot P_{\sigma}(e) & =d_{\sigma} g \cdot \int_{G} \overline{\lambda_{\sigma}\left(g^{\prime}\right)}\left(g^{\prime} e\right) d g^{\prime}=d_{\sigma} \int_{G} \overline{\lambda_{\sigma}\left(g^{\prime}\right)}\left(g g^{\prime} e\right) d g^{\prime} \\
& =d_{\sigma} \int_{G} \overline{\lambda_{\sigma}\left(g^{-1} \tilde{g}\right)}(\tilde{g} e) d \tilde{g}=d_{\sigma} \int_{G} \overline{\lambda_{\sigma}\left(\tilde{g} g^{-1}\right)}(\tilde{g} e) d \tilde{g} \\
& =d_{\sigma} \int_{G} \overline{\lambda_{\sigma}\left(g^{\prime}\right)}\left(g^{\prime} g e\right) d g^{\prime}=P_{\sigma}(g e) .
\end{aligned}
$$

In 2.6, we defined the algebra homomorphism $U: M(G) \rightarrow L(E)$.

Let $E_{\sigma}=P_{\sigma}(E)(\sigma \in \hat{G})$. From 3.3(b) and (c), we see that $E_{\sigma} \cap E_{\tau}=\{0\}$ $(\sigma \neq \tau)$, and $G \cdot E_{\sigma}=E_{\sigma}$.

3.4. Proposition. (a) $E_{\sigma}$ is closed.

(b) The weak direct sum $\bigoplus_{\sigma \in \hat{G}} E_{\sigma}$ is dense in $E$.

(c) $P_{\sigma}(e)=0$ for all $\sigma \in \hat{G}$ iff $e=0$.

Proof. (a) If $\left(e_{n}\right)$ is a net in $E_{\sigma}$ with $e_{n} \rightarrow e \in E$, then $P_{\sigma}(e)=\lim _{n} P_{\sigma}\left(e_{n}\right)$ $=\lim _{n} e_{n}=e$.

(b) Write $\overline{\mathcal{T}_{\sigma}(G)}$ for $\left\{\bar{\phi} \mid \phi \in \mathcal{T}_{\sigma}(G)\right\}$. It follows from 2.8(c) that, if $\phi \in{\overline{\sigma_{\sigma}}}_{\sigma}(G)$, then $\phi * d_{\sigma} \bar{\lambda}_{\sigma}=d_{\sigma} \bar{\lambda}_{\sigma} * \phi=\phi$. Since $U$ is a *-homomorphism, one has $U(\phi) P_{\sigma}=P_{\sigma} U(\phi)=U(\phi)$ (we used the fact that $U\left(d_{\sigma} \bar{\lambda}_{\sigma}\right)=P_{\sigma}$, which is immediate from the definitions). We now reason as follows. Let $S=$ $\operatorname{cls} \bigoplus_{\sigma \in \hat{G}} E_{\sigma}$, and let $\eta \in E^{\prime}$ be such that $\left.\eta\right|_{S}=0$. Fix $\sigma \in \hat{G}$ and $e \in E$; then for all $\phi \in \overline{\mathcal{T}_{\sigma}(G)}$, one has $P_{\sigma} U(\phi) e \in S$, so

$$
0=\left\langle P_{\sigma} U(\phi) \cdot e, \eta\right\rangle=\langle U(\phi) e, \eta\rangle=\int_{G}\langle g e, \eta\rangle \phi(g) d g .
$$

Let $\sigma$ vary over $\hat{G}$; then $\phi$ may be any element of $\cup_{\sigma \in \hat{G}} \overline{\sigma_{\sigma}(G)}$, which has dense linear span in $C(G)(2.8(\mathrm{~b}))$. It follows that $\langle g e, \eta\rangle=0$ for all $g \in G$. Let $g=$ id to see that $\eta=0$. Since $E$ is locally convex, $S=E$.

(c) Suppose $P_{\sigma}(e)=0(\sigma \in \hat{G})$. Then $0=U(\phi) P_{\sigma} e=U(\phi) e\left(\phi \in \overline{\sigma_{\sigma}(G)}\right)$. Repeating part of the proof of (b) shows that $\langle e, \eta\rangle=0$ for each $\eta \in E^{*}$, so $e=0$.

Recalling 2.7, pick $(G, V) \in \sigma$. Let $v_{1}, \ldots, v_{n}\left(n=d_{\sigma}\right)$ be an orthonormal basis for $V$, and let $\left\{u_{i j} \mid 1 \leqslant i, j \leqslant n\right\}$ be the corresponding coordinate functions.

3.5. Lemma. Let $0 \neq e \in E_{\sigma}$. Then there exist finitely many invariant subspaces $\left(B_{j}\right)_{j=1}^{l}, 1 \leqslant l \leqslant d_{\sigma},\left(G, B_{j}\right) \in \sigma$, such that $e \in+_{j=1}^{l} B_{j}$ (the sum, not the direct sum).

Proof. We have $\lambda_{\sigma}(g)=\sum_{j=1}^{n} u_{j j}(g)$. Hence one has $e=P_{\sigma}(e)=$ $d_{\sigma} \sum_{j=1}^{n} U\left(\bar{u}_{j j}\right) \cdot e$. Let $J \subset\{1,2, \ldots, n\}$ be those integers such that $U\left(\bar{u}_{j j}\right) e$ $\neq 0$. Fixing $j \in J$, we will show that $\left\{U\left(\bar{u}_{i j}\right) e \mid 1 \leqslant i \leqslant n\right\}$ spans a subspace $B_{j}$ of the desired type.

We first show that if $g \in G$, then

$$
g \cdot U\left(\bar{u}_{r s}\right)=\sum_{p=1}^{j} u_{p r}(g) U\left(\bar{u}_{p s}\right) .
$$


To see this, let $a \in E, \eta \in E^{*}$, and let $g^{\prime}: E^{\prime} \rightarrow E^{\prime \prime}$ be the adjoint of $g$. Then

$$
\begin{aligned}
\left\langle g \cdot U\left(\bar{u}_{r s}\right) a, \eta\right\rangle & =\left\langle U\left(\bar{u}_{r s}\right) a, g^{\prime} \eta\right\rangle=\int_{G}\left\langle g^{\prime} a, g^{\prime} \eta\right\rangle \bar{u}_{r s}\left(g^{\prime}\right) d g^{\prime} \\
& =\int_{G}\left\langle g g^{\prime} a, \eta\right\rangle \bar{u}_{r s}\left(g^{\prime}\right) d g^{\prime}=\int_{G}\left\langle g^{\prime} a, \eta\right\rangle \bar{u}_{r s}\left(g^{-1} g^{\prime}\right) d g^{\prime} .
\end{aligned}
$$

Now

$$
\bar{u}_{r s}\left(g^{-1} g^{\prime}\right)=\sum_{p=1}^{n} \bar{u}_{r p}\left(g^{-1}\right) \bar{u}_{p s}\left(g^{\prime}\right),
$$

so the equality continues:

$$
\sum_{p=1}^{n} u_{p r}(g) \int_{G}\left\langle g^{\prime} a, \eta\right\rangle \bar{u}_{p s}\left(g^{\prime}\right) d g^{\prime}=\sum_{p=1}^{n} u_{p r}(g)\left\langle U\left(\bar{u}_{p s}\right) \cdot a, \eta\right\rangle .
$$

Comparing first and last terms show that $(*)$ holds.

Recall $j \in J$ is fixed. Let $e_{i}=U\left(\bar{u}_{i j}\right) \cdot e(1 \leqslant i \leqslant n)$. Since $U\left(\bar{u}_{i j}\right) P=$ $P_{o} U\left(\bar{u}_{i j}\right), e_{i} \in E$. Define $W: V \rightarrow B_{j}=\operatorname{span}\left\{e_{1}, \ldots, e_{n}\right\}$ by $W\left(v_{i}\right)=e_{i}$ and by linearity. We compute:

$$
\begin{aligned}
W\left(g v_{i}\right) & =W\left(\sum_{p=1}^{n} u_{p i}(g) v_{p}\right)=\sum_{p=1}^{n} u_{p i}(g) e_{p} \\
& =\sum_{p=1}^{n} u_{p i}(g) U\left(\bar{u}_{p j}\right) \cdot e=g \cdot U\left(\bar{u}_{i j}\right)(e)
\end{aligned}
$$

by (*). The last term is $g \cdot e_{i}=g W\left(v_{i}\right)$; we conclude that $B_{j}$ is $G$-invariant and that $W$ intertwines $(G, V)$ and $\left(G, B_{j}\right)$. Since $e_{j} \neq 0,\left(G, B_{j}\right) \in \sigma$.

Apply the above analysis to each $j \in J$. We obtain subspaces $B_{j} \subset E_{\sigma}$ such that $\left(G, B_{j}\right) \in \sigma$. Any two of these subspaces either coincide or intersect in $\{0\}$ by irreducibility. Form the $\operatorname{sum} B=+_{j \in J} B_{j}$; then $e=\Sigma_{j \in J} U\left(\bar{u}_{j j}\right) \cdot e \in$ $B$.

Lemma 3.5 shows that if $E_{\sigma} \neq 0$, then $E$ contains "many" irreducible subspaces. We can restate this as follows.

3.6. Proposition. If $E_{\sigma} \neq 0$, there is a collection $\left(B_{i}\right)_{i \in I}$ of $G$-invariant subspaces of $E_{\sigma}$, with $\left(G, B_{i}\right) \in \sigma$, such that $+_{i \in I} B_{i}=E_{\sigma}$ (the weak sum, not its closure!).

Proof. Let $M$ be the set of all collections $\left(B_{i}^{\prime}\right)_{i \in I^{\prime}}$ of distinct subspaces of $E_{\sigma}$ satisfying $\left(G, B_{i}^{\prime}\right) \in \sigma$; partially order $M$ by inclusion. By Lemma 3.5, $M \neq \Phi$. Clearly we can zornify; let $\left(B_{i}\right)_{i \in I}$ be maximal in $M$. Since the $B_{i}$ are distinct and irreducible, $B_{i} \cap B_{j}=\{0\}(i \neq j)$. We now define $B=+{ }_{i \in I} B_{i}$. If $e \in E_{\sigma}, e \notin B$, perform the construction of 3.5 to obtain subspaces $C_{j}$, $1 \leqslant j \leqslant l$, such that $\left(G, C_{j}\right) \in \sigma$. At least one $C_{j_{0}}$ is not in $\left(B_{i}\right)_{i \in I}$, for otherwise $e \in+{ }_{j=1}^{l} C_{j} \subset+_{i \in I} B_{i}$. Add $C_{j_{0}}$ to $\left(B_{i}\right)_{i \in I}$ to obtain a contradiction. 
3.7. Proposition. $E_{\sigma}$ is the smallest closed subspace of $E$ containing all $G$-invariant subspaces $B$ such that $(G, B) \in \sigma$.

Proof. Let $B \subset E,(G, B) \in \sigma$, and let $V, v_{1}, \ldots, v_{n}, u_{i j}$ be as in 3.5. Let $W: V \rightarrow B$ be intertwining, $e_{i}=W\left(v_{i}\right)$. Then

$$
\begin{aligned}
P_{\sigma}\left(E_{i}\right) & =d_{\sigma} \int_{G}\left(g e_{i}\right) \overline{\left(\lambda_{\sigma}(g)\right)} d g=d_{\sigma} \int_{G} g \cdot W\left(v_{i}\right) \overline{\lambda_{\sigma}(g)} d g \\
& =d_{\sigma} \int_{G} W\left(g v_{i}\right) \overline{\lambda_{\sigma}(g)} d g=W\left(\int_{G}\left(g v_{i}\right) \overline{\lambda_{\sigma}(g)} d g\right) .
\end{aligned}
$$

The last step uses $2.5(\mathrm{c})$ and the fact that $W$ is continuous as a map from $V$ to $E$. The equality continues:

$$
\begin{aligned}
& =W\left[d_{\sigma} \int_{G}\left(\sum_{p=1}^{n} u_{p i}(g) v_{p}\right) \cdot\left(\sum_{r=1}^{n} \overline{u_{r r}(g)}\right) d g\right] \\
& =W\left[d_{\sigma} \sum_{p=1}^{n}\left(\int_{G} \sum_{r=1}^{n} u_{p i}(g) \overline{u_{r r}(g)} d g\right) v_{p}\right] \\
& =W\left[d_{\sigma} \sum_{p=1}^{n} \frac{1}{d_{\sigma}} \delta_{p i} v_{p}\right]=W\left(v_{i}\right)=e_{i} .
\end{aligned}
$$

We have used the orthogonality results for the $u_{i j}$ 's (2.8(a)). This shows that $P_{\sigma} e_{i}=e_{i}$; i.e., $e_{i} \in E$. Thus $B \subset E_{\sigma}$, so $E_{\sigma}$ contains all invariant subspaces such that $(G, B) \in \sigma$. Lemma 3.5 shows that $E_{\sigma}$ is the smallest such.

3.8. REMARK. The proofs of $3.4,3.5$, and 3.7 are straightforward generalizations of portions of the proof of 27.44 in [2].

3.9. Theorem. Let $E \neq\{0\}$ be topologically irreducible. Then $(G, E) \in \sigma$ for some $\sigma \in \hat{G}$. In particular, $E$ has finite dimension.

Proof. Since $E \neq\{0\}$, there exists $\sigma \in \hat{G}$ such that $P_{\sigma} \neq 0$ (3.4(c)). Consider one such $\sigma$; the space $E_{\sigma}$ is invariant and closed, hence $E_{\sigma}=E$. Let $e \in E_{\sigma}$; by $3.5, e \in \bigoplus_{j=1}^{l} B_{j}$, where each $\left(G, B_{j}\right) \in \sigma$. By finite dimensionality $+{ }_{j=1}^{l} B_{j}$ is closed; it follows easily that $l=1$ and that $(G, E) \in \sigma$.

3.10. Corollary. Let $E^{\prime} \subset E$ be topologically irreducible. Then $E^{\prime} \subset E_{\sigma}$ for some $\sigma \in \hat{G}$.

Proof. Since $\left(G, E^{\prime}\right) \in \sigma$ for some $\sigma(3.9), E^{\prime} \subset E_{o}$ (3.7).

\section{REFERENCES}

1. N. Bourbaki, Éléments de mathématique. Fasc. XIII. Livre VI: Integration, Chaps. 1-4, 2nd ed., Actualités Sci. Indust., no. 1175, Hermann, Paris, 1965. MR 36 \#2763.

2. E. Hewitt and K. Ross, Abstract harmonic analysis, vol. I, Springer-Verlag, Berlin; Academic Press, New York, 1963; vol. II, Springer-Verlag, Berlin and New York, 1970. MR 28 \# 158; 41 \# 7378; erratum, 42, p. 1825.

3. G. Warner, Harmonic analysis on semi-simple Lie groups. I, Springer-Verlag, New York, 1972.

Department of Mathematics, University of Wisconsin-Parkside, Kenosha, Wisconsin 53140 